\title{
Review of: "Reliable evaluation method of heating power of magnetic nanofluids to directly predict the tumor temperature during hyperthermia"
}

\author{
Kuo-Chi Liu \\ 1 Far East University, Taiwan
}

Potential competing interests: The author(s) declared that no potential competing interests exist.

This paper aims to establish a pseudo-tumor environment system to measure the heating power of magnetic nanofluids (MNs) in vitro to accurately predict AC heat-induced performance in tumors during nanofluidic hyperthermia. The experimental data are used to verify the feasibility of the pseudo-tumor environment system. This research will contribute to the development of magnetic hyperthermia and is a topic worthy of further exploration. However, there are doubts about the reliability of the current results:

1. In magnetic hyperthermia, the nano-magnetic particles are mixed in the tumor tissue and not exist alone, and the effect of the alternating magnetic field on the nano-magnetic particles is not necessarily equivalent to the current method.

2. Although the current results have been verified by experimental data, the experimental object is placed on the temperature control board, and the overall temperature of the experimental object is well controlled.

3. Although the main component of the organism is water, the direct replacement of peri-tumor environmental tissue with conditioned water is debatable. Basically, the penetration of the AC magnetic field and the heat transfer properties of the tissue will be altered.

Also, this paper has been published. Peer review is no longer relevant for published papers. 